\title{
Design and Implementation of the Logistics Management System Based on J2EE Technology
}

\author{
Yiming $\mathrm{Li}^{1, \mathrm{a}}$, Lan $\mathrm{Bai}^{2}$, Liang Wang ${ }^{3}$, Huan Zhang ${ }^{4}$ \\ ${ }^{1,2,3,4}$ School of business, Hebei agriculture university, Hebei, China \\ aliyiminghbnd@163.com
}

Keywords: logistics management system ; J2EE; JSP

\begin{abstract}
The J2EE model is the use of advanced software layered design ideas to support framework-based development, and it can reduce development effort and cost, while reducing the degree of coupling of components, and greatly enhance software maintainability, scalability to meet the large-scale management information system requirements. The paper proposes the design and implementation of the logistics management system based on J2EE technology. This article describes the basic functions of logistics management system based on J2EE technology design, the overall structure and implementation of key technologies involved in the system.
\end{abstract}

\section{Introduction}

As global economic integration, China has gradually become the world's manufacturing and procurement centers, logistics increasingly important role in them. In order to meet the needs of modern economic development, logistics enterprises to information technology, integrated, professional change, fully integrated logistics and information technology, the establishment of a logistics management system based on modern management information network architecture.

Namely Java 2 Enterprise Edition J2EE, Sun has introduced a multi-layered structure of the distributed application model, the model has the ability of reusable components, based on the Extensible Markup Language (XML) data exchange, a unified security model, and flexible transaction control, it set up with scalability, flexibility, ease of maintenance of enterprise information systems to provide a good mechanism, has incomparable advantages compared with traditional Internet application model [1]. Developers can not only quickly new problem domain solutions, and its platform-independent, component-based J2EE solution is no longer subject to the restrictions of providers of products and application programming interface.

J2EE architecture, the presentation logic, business logic and data logic, phase separation, and the use of advanced software layered design ideas, support based on framework development, reduce development effort and cost, while reducing the degree of coupling of components, greatly enhancing software maintainability, scalability, and to meet the requirements of large-scale management information system.

With the rapid development of the current socio-economic, manufacturing, commerce, and the size of the business continued to expand, products, raw materials, a large demand, the amount of logistics is also increasing. Therefore, the demand for third-party logistics services is virtually gone up. To further enhance the efficiency of modern logistics management, logistics management must have a thorough improvement. In fact, whether at home or abroad, logistics management information integration model has long been widely recognized and has been widely applied. However, specifically how to do to make the information system and management structure is not out of line and keep up with the pace of upgrading it? This is an urgent need for more in-depth study of the modern enterprise logistics management information system, in order to allow enterprises to achieve sustainable development goals at the same time, as much as possible to maximize the quality services to customers; the same time, in the service process, but also seek to reduce logistics costs, reducing costs, and ultimately achieve the purpose of profit. 
With the development of J2EE gradually evolved into component-based, modular components, the center of gravity of the developer from learning techniques to familiar with business, business infrastructure platform that can help to achieve and to build fast and flexible business systems. Engaged in the root of customer needs-based, starting from the fundamental task of the software development, to find and use some kind of description of the business method, so this method can adapt to various changes in the business infrastructure platform. For many years engaged in enterprise management information system, application, in-depth understanding of customer needs, summed up the industry business model, and workflow system, rights management systems, reporting and management system based on J2EE technology accumulation re-designed and realized based on J2EEtechnology business infrastructure platform. The paper proposes the design and implementation of the logistics management system based on J2EE technology.

\section{Logistics management information system functions model}

Enterprise management information system, logistics management information system is very important to a subsystem [2]. The main role of the logistics management information system is the logistics system and beyond all the information collection, storage and processing, and extract an effective logistics management information, and then various forms of output. Under normal circumstances, the common form of logistics management information systems: forms, documents, graphics and reports. These outputs form is usually determined based on output, managers and leaders can be a reasonable overall planning of logistics activities based on this information correct and effective organization of work, and then the logistics activities, coordination and control of the normal operating subsystem run, this will reduce the material loss of power, easy to greatly improve the management efficiency of the logistics work. For logistics management and other enterprise management, operational decisions will be more scientific.

Logistics management information system to work, product ordering, purchasing, inventory, shipping and distribution, information management and deployment, you can aim at the timely and accurate, but also of the communication base and network of communication lines, and to protect the modernization of the communication means in order to improve the efficiency of logistics operating system. On the logistics enterprises, the basic logistics activities including transportation, storage, handling, packaging, distribution, processing, logistics management information system also runs through this activity.

Customer management subsystem: customer data collection, classification, archiving, retrieval and management, comprehensive grasp of different customer groups, the nature of the customer, customer demand, customer credit programs, prices, markets, information and other information to provide customers with a variety of services, timely and properly resolve the various problems encountered by customers in cooperation [3]. The subsystem includes a customer login management, customer data management, customer inquiries and other functions, as is shown by equation1.

$$
Z=\left[\begin{array}{l}
\frac{\partial I}{\partial x} \\
\frac{\partial I}{\partial y}
\end{array}\right]\left[\begin{array}{ll}
\frac{\partial I}{\partial x} & \frac{\partial I}{\partial y}
\end{array}\right]=\left[\begin{array}{ll}
g_{x}^{2} & g_{x} \cdot g_{y} \\
g_{x} \cdot g_{y} & g_{y}^{2}
\end{array}\right]
$$

Treasury management process, in order to carry out the reservoir area and the library full range of effective management, it is necessary to achieve the logistics management information network. Otherwise, a library, and storage all by hand, not only the efficiency will be greatly reduced, but also for the staff, because of their workload is very large, tend to do a lot of wasted effort, and thus give rise to twice the power semi-adverse situation.

Order Management subsystem: the order is a basis for settlement of logistics operations and costs, through standardization, modularity and process orders, a reasonable allocation of the implementation details of the logistics services and charges, and as a basis, the allocation of appropriate resources 
monitor the implementation of the results and accounting costs incurred, and the parties in the implementation of the orders be evaluated to obtain the information of customers, costs, funding, delivery of customer service and business sectors as a reference. The subsystem consists of the order reception / entry, order inquiries, order tracking and other functions, as is shown by equation2.

$$
\begin{array}{lll}
\Phi_{j, k, m}(x, y)=\phi_{j, k}(x) \phi_{j, m}(y) & \Psi_{j, k, m}^{1}(x, y)=\phi_{j, k}(x) \psi_{j, m}(y) \\
\Psi_{j, k, m}^{2}(x, y)=\psi_{j, k}(x) \phi_{j, m}(y) & , & \Psi_{j, k, m}^{3}(x, y)=\psi_{j, k}(x) \psi_{j, m}(y)
\end{array}
$$

Speaking from a different angle from vertical management point of view, can the work of the entire logistics enterprise is divided into operating layer, control layer and the management at three levels. Logistics management information of these three levels must also be involved everywhere, exhaustive [4]. Conversely, if the course of business of the three levels of bad problems, then mop-up will adversely affect the overall operation of the logistics enterprises, as is shown by figure1.

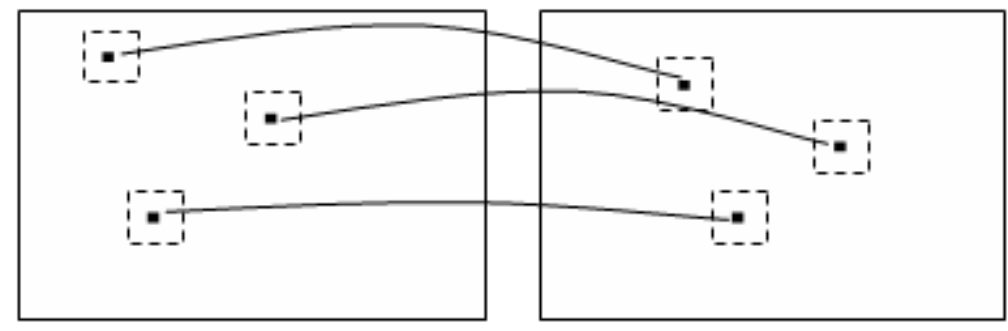

Figure. 1 Logistics management system example

The storage management subsystem: warehouse management, including storage resource management and business processes to achieve, you can achieve a multi-polar management of the Treasury, operating motion segmentation and scheduling strategies, stock management, and inventory of a variety of strategies [5]. Including receiving, quality inspection, put away, picking, distribution processing, shipping, replenishment, inventory control, visualization, monitoring, transfer database, inventory and other functions, as is shown by equation 3 .

$$
X^{\prime}=W(X, P)=\left(\begin{array}{ccc}
a_{1} & a_{2} & d_{1} \\
a_{3} & a_{4} & d_{2} \\
0 & 0 & 1
\end{array}\right)\left(\begin{array}{l}
x \\
y \\
1
\end{array}\right)
$$

Supplier management, according to the history of dealings with suppliers, suppliers, qualification management, and regularly update the product catalog of supplier, seamless integration with the contract module, query the information of the contract signed with the supplier and performance of the contract.

\section{Design and implementation of the logistics management system based on J2EE technology}

Design and implementation of logistics management system based on J2EE business infrastructure platform, the J2EE business infrastructure platform has been successfully applied to the design and development of multiple systems, and applied to the human resources management system, customer service systems, decision support system software has the following characteristics: based on open architecture, based on the MVC structure designed to achieve the separation of business and data. Advanced architecture and design ideas, different people develop different business modules. System with easy to maintain, adapt to a variety of heterogeneous and interoperability. Business functions with the improvement of market structure, mode, and operating rules may at any time to expand and upgrade.

Hibernate is an excellent open-source ORM (Object Relational Mapping) framework, which provides a powerful, high performance object to relational database persistence services. Hibernate, Java programmers can use object-oriented programming thinking to manipulate the database, 
significantly reducing the time of hand-coding operations, JDBC and SQL [6]. In addition, Hibernate supports almost all major commercial databases and open source database, and supports a variety of caching mechanism, through a simple configuration, the user can use the services of third-party cache, greatly improving the efficiency of the operation of the system database. The data persistence layer using is Hibernate + DAO pattern of development.

This architecture, each layer is only dependent on its layer below, for example, changes to the database without modifying the presentation layer; something unique in each layer to other layers hidden, so to ensure the application to modify them easy to modify not cascade to other layers. This multi-tier architecture is particularly important in the enterprise-class system, it ensures a clear division of responsibilities, with greater scalability and flexibility is defined as equation 4.

$$
\sum_{X \in \Omega_{i}}\left(\frac{\partial I}{\partial W} \frac{\partial W}{\partial P}\right)^{T}\left[I(W(X, P))+\frac{\partial I}{\partial W} \frac{\partial W}{\partial P} \Delta P-I_{k-1}(X)\right]=0
$$

Servlet is a server-side Java program is built on the cornerstone of Web-based application, is essentially a small, platform-independent Java classes, with the characteristics of cross-server platform. The servlet receives the request object from the extracted parameters to handle the business logic, and ultimately respond.

JSP (Java Server Pages) is initiated by Sun, a number of companies participate in the development of a powerful dynamic web technology standards. JSP provides a unique development environment for creating highly dynamic Web applications. A JSP Java as its scripting language, to join the Java program fragment and JSP tag in the HTML file, JSP has all the advantages of the Java language, including security, cross-platform; JSP technology page design and business logic separation to distinguish between the role of Web designers and Web developers. In the presentation layer of the system, and ultimately to the client's view is generated by JSP, the JSP and custom tag library extension, as is shown by equation 5 .

$$
\begin{aligned}
f_{j+1}(x, y) & =\sum_{k, m} C_{j+1, k, m} \Phi_{j+1, k, m} \\
& =\sum_{k, m} C_{j, k, m} \Phi_{j, k, m}+\sum_{k, m} D_{j, k, m}^{1} \Psi_{j, k, m}^{1}+\sum_{k, m} D_{j, k, m}^{2} \Psi_{j, k, m}^{2}+\sum_{k, m} D_{j, k, m}^{3} \Psi_{j, k, m}^{3}
\end{aligned}
$$

XML (extensible Markup Language, Extensible Markup Language) is a content-oriented markup language created by the $\mathrm{W} 3 \mathrm{C}$. Because it has a more semantic, good scalability and easy to use, self-describing characteristics particularly suitable for semi-structured data on the Web, it can be used for data exchange [7]. The implementation of this system, the server required standard JAVA deployment descriptor, the data persistence layer of the Hibernate configuration file, and use XML mapping document and the system required some configuration.

J2EE is to develop scalable, ideal load balancing, multi-tier distributed cross-platform enterprise application platform. The heart of J2EE is a set of norms and guidelines for the definition of a standard platform for developing multi-tier distributed enterprise applications using the Java language, J2EE effectively meet the deepening needs of industry information. XML technology, $\mathrm{XML}$ is a meta-markup language, in the construction of information systems as a data carrier and medium of exchange, to maintain the openness and ease of integration of the system data.

Open source frameworks (eg Struts, Log4j, etc.) as the information system of the Web framework, the framework is based on J2EE and enterprise-level Web application framework comply with MVC2, its core is to achieve loose coupling of multi-tier application for the business group, the platform laid a good foundation. Web framework used in the corporate area of expertise is still limited, the need for a Web framework, the corresponding expansion of the following: (1) Extending Web Framework core components, as well as data validation components, increased exception handling components and logging utility class. (2) expansion of the tag library, including the drop-down data source label, tree control label, date and time format of the control tag, the page permissions tab and page elements, access control labels, navigation tabs on the location of the page, as well as abnormal display and labels. 
Increase access to the framework of process management, graphics management, and unified security management, report management utility class. For example, the process management utility class used to implement the generated process instance, the process pass (including the normal transfer and jump transfer) process view, monitor the process flow and other functions, and provide page customization utility, according to the data set, the format parameters automatically generated JSP files.

Data sets defined components, the operating components of data sets loaded components, data sets. The data set defined components to define data query, stored procedure mapping, data queries, stored procedure defined to store the XML document property is set to include the code columns, column names, data types, extended attributes, the main foreign key, the update flag. Data set to load components will be stored in XML for dynamic loading, complete the two-dimensional table in the relational database and object-oriented systems object mapping process [8]. Operating components of the data set encapsulates the data access details on the background of the relational database application to access the system through a data set of operating components, both abstract data where the data source, and abstract database CRUD (Create, Replace, Update, Delete) logic., as is shown by equation6.

$$
\Delta P=H^{-1} \sum_{X \in \Omega_{i}}\left[\frac{\partial I}{\partial W} \frac{\partial W}{\partial P}\right]^{T}\left[I_{k-1}(X)-I(W(X, P)]\right.
$$

Application J2EE technology design and implementation of logistics management system using B / S multi-tier architecture, so that application data is limited in the corresponding layer, reducing the coupling between the layers of data, to improve the overall framework can maintain and scalability. Windows XP operating system using Visual VB.net to achieve the above rule set and computing algorithm. For randomly generated data sets, the relationship between the probabilities of $85 \%$, we do the extended test, the number of objects in increments of 23 , recorded by the sub-formal context. The paper proposes the design and implementation of the logistics management system based on J2EE technology, the results shown in Figure 2.

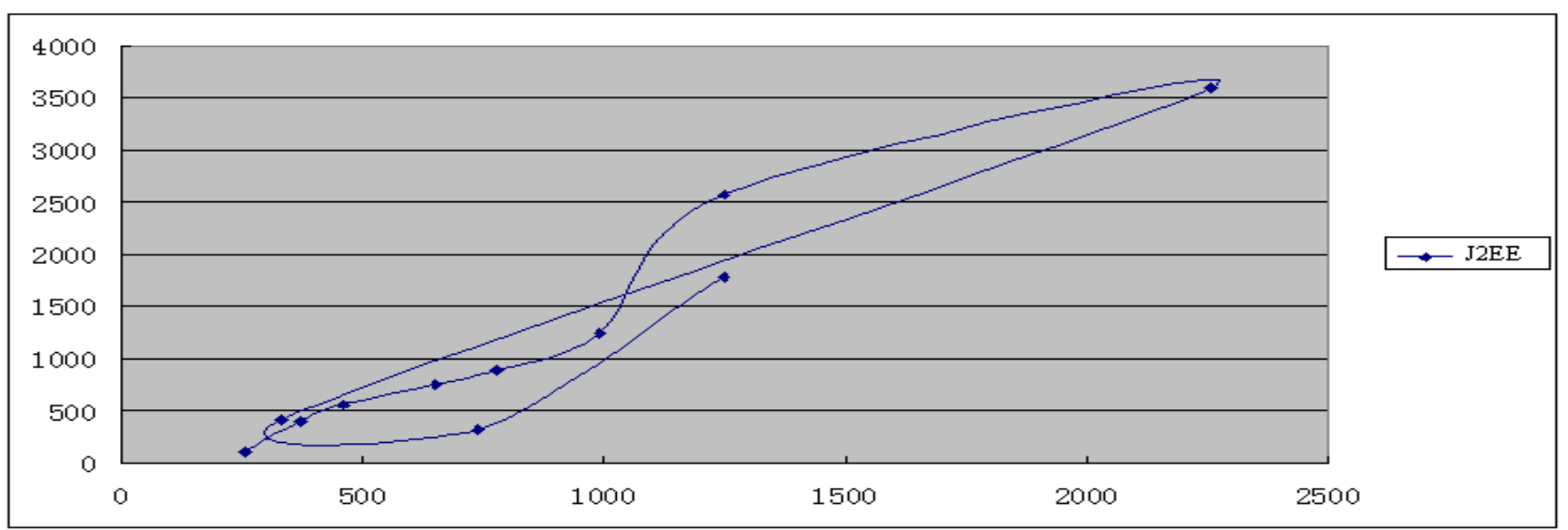

Figure. 2 The design and implementation of the logistics management system based on J2EE technology map

Business infrastructure platform and customer management and business are more common infrastructure platform for application software development. Interaction between software and hardware, and management of operating system platform, database platform, J2EE infrastructure platform to solve the interaction between system software and operating system platforms and management issues, J2EE business infrastructure platform to solve a business description of the application software and operating system platforms, the interaction between the management of the database platform, J2EE infrastructure platform. 
In this paper, on the basis of analysis of enterprise management information system "open, interconnected, integrated and practical needs, the logistics management system of the J2EE business infrastructure platform-based design and implement solutions. Described the key technologies of the system is involved in the J2EE technology web application framework, workflow platform, and rights management platform, report management platform, and introduced the system of functional modules and features.

\section{Summary}

The paper proposes the design and implementation of the logistics management system based on J2EE technology. Logistics Management System Design and Implementation of J2EE business infrastructure platform based on the hierarchical structure of the software platform, J2EE business infrastructure platform provided by the business component, software services, application and database platform, J2EE infrastructure platform isolation improve software reusability, can improve the efficiency of the development, system development and application of the key.

\section{References}

[1] Liu Guangli, Lou Zhenlin, Hou Yunxian, Shen Cuihua, "Rural Emergency System Based on WebGIS", JCIT, Vol. 6, No. 2, pp. $342 \sim 346,2011$

[2] WeiHua Li, "The Research on the Emergency Logistics Risk Analysis with incomplete information", IJACT, Vol. 4, No. 5, pp. $304 \sim 310,2012$

[3] Wenlong Du, "Evaluation System for Simulation Model of City Logistics Planning", JCIT, Vol. 7, No. 8, pp. $169 \sim 176,2012$.

[4] Wei Li, "Research on Logistics Financial Credit Evaluation of Third-Part Logistics Enterprises with Uncertain Information", AISS, Vol. 4, No. 8, pp. $171 \sim$ 177, 2012.

[5] LIU Li-zhuo, "Analysis of the Relationship Between QinHuangDao Port Logistics and Economic Growth", AISS, Vol. 4, No. 4, pp. $105 \sim 114,2012$

[6] Yuran Jin, "An Improved Profit Distribution Model for Iron and Steel Logistics Alliance", AISS, Vol. 4, No. 3, pp. $159 \sim 166,2012$

[7] Sui Cui, Ding Jingzhi, "Business Orientation of Logistics Development for State Reserve Warehouse in China", AISS, Vol. 3, No. 6, pp. $167 \sim$ 173, 2011

[8] Li Hanqing, Ru Yihong, Han Jihua, "Emergency Logistics Systems Synnergetics Research", AISS, Vol. 3, No. 6, pp. $245 \sim 250,2011$ 